\title{
Case Report: Propranolol increases the therapeutic response to temozolomide in a patient with metastatic paraganglioma
}

\section{[version 1; peer review: 2 approved]}

\author{
Miguel-Angel Díaz-Castellanos¹, Karina Villar Gómez de las Heras²,3, \\ Tamara Díaz-Redondo4, Encarnación González-Flores5 ${ }^{5}$ Virginia Albiñana6, \\ Luisa-María Botella (D)6 \\ ${ }^{1}$ PHEiPAS, Castellón de la Plana, 12006, Spain \\ ${ }^{2}$ Alianza VHL, Centro Cívico Rogelio Soto, Sabadell, 08204, Spain \\ ${ }^{3}$ Sanidad Castilla-La Mancha, Toledo, Spain \\ 4Servicio de Oncología, Hospital Universitario Carlos Haya, Málaga, 29010, Spain \\ ${ }^{5}$ Servicio de Oncología, Hospital Universitario Virgen de las Nieves, Granada, 18014, Spain \\ ${ }^{6}$ Centro de Investigaciones Biológicas, U-707 CIBERER, Madrid, Spain
}

V1 First published: 04 Dec 2017, 6:2087
https://doi.org/10.12688/f1 000research.13185.1

Latest published: 04 Dec 2017, 6:2087

https://doi.org/10.12688/f1000research.13185.1

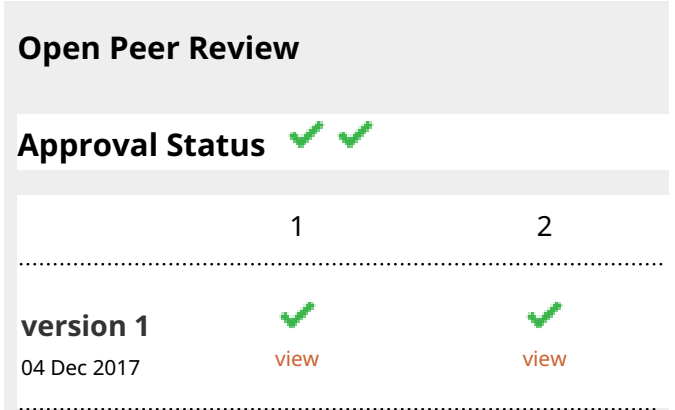

1. Angeles Juarranz, Autonomous University of Madrid, Madrid, Spain

2. Carlos Jara ID, King Juan Carlos University (URJC), Madrid, Spain

Any reports and responses or comments on the article can be found at the end of the article.

Keywords

Paraganglioma, rare oncologic diseases, repurposing drugs, pseudohypoxic cancer syndromes 
Corresponding author: Luisa-María Botella (cibluisa@cib.csic.es)

Author roles: Díaz-Castellanos MA: Conceptualization, Data Curation, Supervision, Validation, Visualization, Writing - Review \& Editing; Gómez de las Heras KV: Conceptualization, Data Curation, Supervision, Writing - Review \& Editing; Díaz-Redondo T: Conceptualization, Investigation, Methodology; González-Flores E: Conceptualization, Formal Analysis, Investigation, Methodology; Albiñana V:

Conceptualization, Investigation, Validation; Botella LM: Conceptualization, Validation, Writing - Original Draft Preparation, Writing Review \& Editing

Competing interests: No competing interests were disclosed.

Grant information: The author(s) declared that no grants were involved in supporting this work.

Copyright: @ 2017 Díaz-Castellanos MA et al. This is an open access article distributed under the terms of the Creative Commons Attribution License, which permits unrestricted use, distribution, and reproduction in any medium, provided the original work is properly cited.

How to cite this article: Díaz-Castellanos MA, Gómez de las Heras KV, Díaz-Redondo T et al. Case Report: Propranolol increases the therapeutic response to temozolomide in a patient with metastatic paraganglioma [version 1; peer review: 2 approved] F1000Research 2017, 6:2087 https://doi.org/10.12688/f1000research.13185.1

First published: 04 Dec 2017, 6:2087 https://doi.org/10.12688/f1000research.13185.1 


\section{Introduction}

Hereditary paraganglioma and pheochromocytoma syndromes (HPP) are characterized by rare tumors called pheochromocytomas (PHEOs) and paragangliomas (PGLs). These are highly vascularized catecholamine-secreting tumors of neural crest origin (chromaffin cells derived from the adrenal medulla - pheochromocytoma - or extra-adrenal paraganglia - paragangliomas), distributed symmetrically along the paravertebral axis. Although tumors are usually histologically benign, most of them hyper-secrete catecholamines, causing high cardiovascular morbidity and mortality ${ }^{1}$, and symptoms related to mass effect. If left untreated, a subset of these tumors will metastasize in bones, lungs, liver or lymph nodes ${ }^{2}$. These patients can develop other rare tumors too, such as renal cancer and gastrointestinal stromal tumors. However, tumorigenesis is rare in the first decade of life.

HPP syndromes are due to pathogenic variants of genes, SDHx genes, a group of multiple nuclear genes encoding subunits of the succinate dehydrogenase (SDH) enzyme complex. Similarly to other autosomal-dominant hereditary cancer disease - von Hippel-Lindau (VHL) -paraganglioma-pheochromocytoma (PGL/ PCC) syndrome is related to the hypoxia pathway ${ }^{3}$. Hypoxiainducible factor 1 (HIF1) regulates the cellular response according to oxygen levels. HIF is a heterodimer of a HIF1- $\alpha$ subunit, regulated by oxygenation and a $\beta$ subunit. Under normal oxygen conditions, HIF1- $\alpha$ degradation is promoted by the E3 ubiquitin ligase pVHL (von Hippel Lindau factor). In lower oxygen cell environments, VHL recognition of HIF- $1 \alpha$ is decreased, allowing HIF1 to promote cellular survival and growth ${ }^{4}$. In cancer, the hypoxia pathway is switched by the tumoral cells leading to the tumor growth ${ }^{5}$. Pseudo-hypoxic states are those leading to the hypoxia-pathway gene expression, under normoxic conditions.

Germline mutations in the onco-suppressor gene, called $\mathrm{Vhl}$ leads to the autosomal-dominant hereditary cancer disease VHL. VHL disease is clinically characterized by retinal and CNS hemangioblastomas, RCC (renal clear cell carcinoma), PCC, neuroendocrine pancreatic tumors and pancreatic cystadenomas, endolymphatic sac tumors and broad ligament cystadenomas. Closely related to this disease are the mutations involving the succinate dehydrogenase genes (SDHx genes) ${ }^{6}$. The lack of function of SDHx genes leads to an inhibition of prolyl hydroxylases by alpha-ketoglutarate. These hydroxylases "target" HIF $1 / 2-\alpha$ by specific hydroxylation to be recognized and bound by VHL. In this way, hydroxylases cannot help in the degradation of HIF, and a state of pseudo-hypoxia ${ }^{7}$ is created. Thus, pseudo-hypoxic states display similar hypoxia-pathway triggered gene expression, but under normoxic conditions.

Surveillance recommendations for both diseases (VHL disease and PHEO/PGL) ${ }^{2}$ are very similar. It is important to suspect, localize and resect these tumors.

The present case presents the clinical evolution and management of a patient with a hereditary paraganglioma syndrome. The combination of high doses of the beta blocker propranolol ( $3 \mathrm{mg} / \mathrm{Kg} /$ day) and the DNA intercalating agent, temozolomide, was been successful in the treatment of the SDHA metastatic paraganglioma.

\section{Case description}

A 53 year old man, with personal and family history of hypertension, was diagnosed with a $4 \mathrm{~cm}$ tumoral mass at costovertebral D6-D7 with a 21 max SUV, during a routine check-up in March 2010. The only manifestation of disease was elevated blood pressure. He was being treated at this time with irbesartan/ hydrochlorothiazide $(1250 \mathrm{mg} / 12.5 \mathrm{mg}$, respectively). After surgery, the pathology diagnosis was of PGL with a Ki67 around 5-8\%, and synaptophysin and chromogranin positive. Genetic analysis found a mutation in the SDHA gene, leading to a heterozygous missense p.Ser445Leu change, defined as pathogenic ${ }^{8}$. The patient remained without treatment and symptom-free until August 2014, when a back pain appeared around the D10 region, with progressive worsening. In October, as the pain persisted, he underwent MRI and 18F-DG-PET scan and was diagnosed with several metastasis at: D6-D7, right acetabulum $(3 \mathrm{~cm})$; vertebral hemi-body D10 $(3 \mathrm{~cm})$; vertebral body of D12 $(2 \mathrm{~cm})$; and left iliac crest $(1 \mathrm{~cm})$. A scintigraphy with Indium-111 pentetreotide suggested metastatic disease of probably neuroendocrine origin. D10 lesion biopsy confirmed the diagnosis of PGL metastasis with a Ki67 around 15-20\% (Figure 1A). The patient was treated with doxazosin ( $8 \mathrm{mg} /$ day) and bisoprolol (10 $\mathrm{mg} /$ day $)$.

In November 2014, the patient started on systemic treatment with denosumab (120 mg every 28 days) and lanreotide $(120 \mathrm{mg}$ every 14 days). After 4 months, a PET-TAC showed a metabolic stabilization of the disease, but with a slight growth of lesions according to MRI (Figure 1B). D6 and D10 lesions were then treated by radiotherapy (Cyberknife). To this purpose, in March/ April 2015 the patient underwent SBRT with CiberKnife (24 Gy in D10, 15 Gy in D12 and 35 Gy in D6-7), and the pain disappeared. After 6 months, the D7 and D12 lesions were also irradiated and completed SBRT with CiberKnife (24 Gy in left iliac bone and 24 Gy to right acetabulum).

Response to radiotherapy was good, showing stability of lesions and decrease of max SUV. No new lesions appeared. In June 2015, a thermoablation and right acetabulum metastasis cementation was performed with good results.

At this moment, the anti-hypertensive bisoprolol was changed to propranolol(120mg/day), due to preliminary reports of propranolol's good results in VHL tumors'. During the following 11 months, the patient remained stable without disease progression (Figure 1C). Lanreotide dose was decreased to $120 \mathrm{mg}$ every 3 weeks, due to side effects. Side effects included nausea, vomits, diarrhea and hyperglycaemia.

In May 2016, metanephrines and chromogranin were raised above normal levels, as follows: Chromogranin A, $106 \mathrm{ng} / \mathrm{mL}$ (normal level, <93); urine fractionated Norepinephrine, $196 \mathrm{mcg} / 24 \mathrm{~h}$ (normal levels, 15-80); urine fractionated normetanephrine, $1316 \mathrm{mcg} / 24 \mathrm{~h}$ (normal levels, 128-484); urine total metanephephrine, $1394 \mathrm{mcg} / 24 \mathrm{~h}$ (normal levels, 220-680). A 18FDG-PET-TAC revealed an important metastatic dissemination all over the skeleton, showing more than 40 small new lesions less than $2 \mathrm{~cm}$, and with scarce or null Octreoscan and ${ }^{123} \mathrm{I}-\mathrm{MIBG}$ uptake (Figure 1D). 

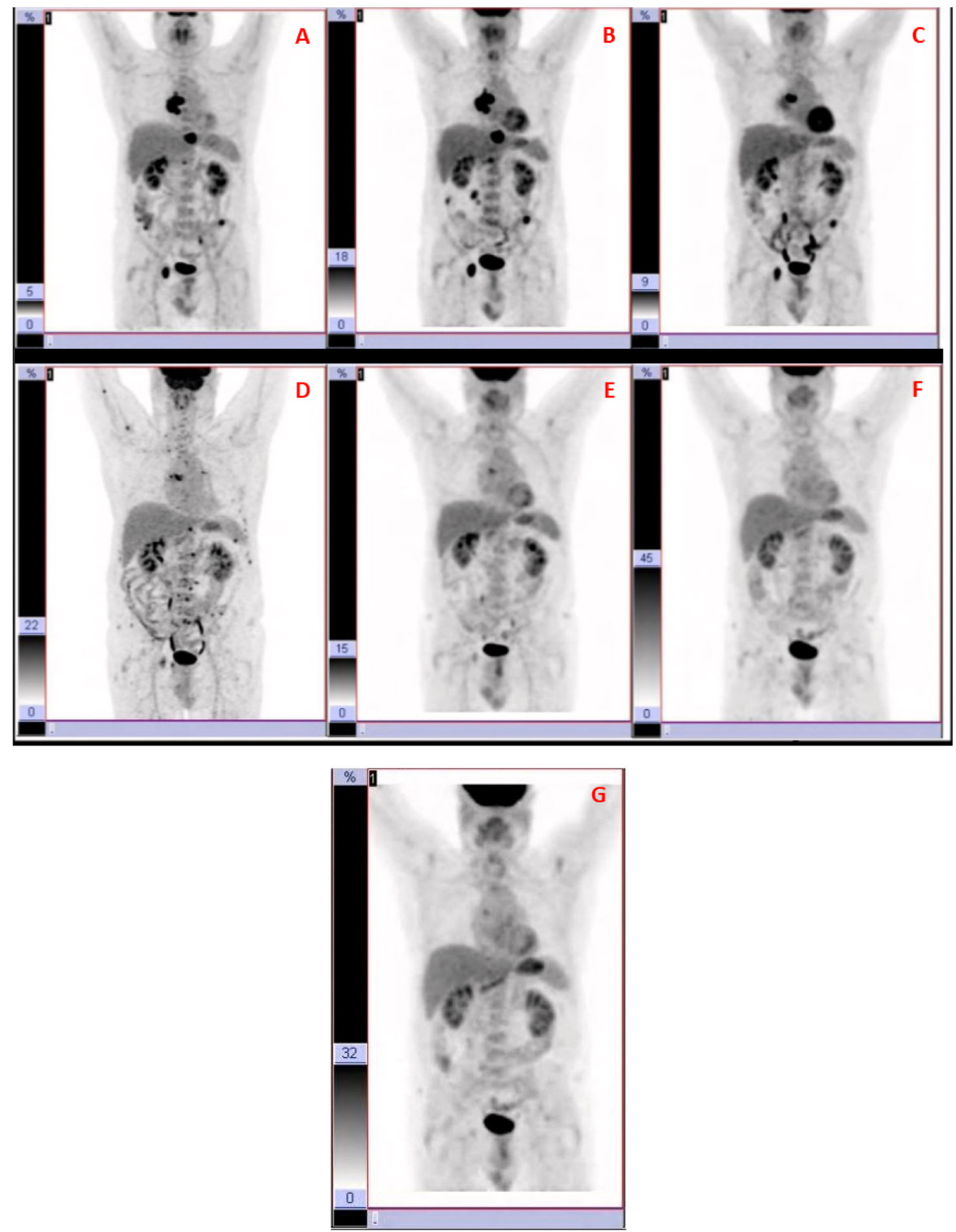

Figure 1. MRI and 18F-DG-PET scan. (A) In October 2014, several metastasis at D6-D7, right acetabulum (3cm); vertebral hemi-body D10 $(3 \mathrm{~cm})$; vertebral body of D12 $(2 \mathrm{~cm})$; and left iliac crest $(1 \mathrm{~cm})$ were observed. (B) 4 months later, a PET-TAC showed a metabolic stabilization of the disease, but with a slight growth of lesions according to MRI. (C) During the following 11 months, the patient remained stable without disease progression. (D) In May 2016, a 18 FDG-PET-TAC revealed an important metastatic dissemination all over the skeleton, showing more than 40 small new lesions $\left(<2 \mathrm{~cm}\right.$ ), and with scarce or null Octreoscan and ${ }^{123}$ |-MIBG uptake. (E) In December 2016, most of lesions had disappeared according to PET-TAC. (F) In July 2017, a new PET supported the previous results, showing the remaining two lesions but with maximum SUV lower. (G) In October 2017, 4 months since the last dose of temozolomide, and propranolol as the only treatment (240mg/day), the disease remains controlled according to this last PET-TAC. 
From this moment on, a combined treatment of temozolomide $\left(75 \mathrm{mg} / \mathrm{m}^{2}\right.$ for 21 days every 28 days) and an increase of propranolol up to $3 \mathrm{mg} / \mathrm{kg} /$ day $(240 \mathrm{mg} /$ day) was prescribed. After the first cycle of temozolomide, results were evaluated in August 2016 at National Institutes of Health, where the previously detected metastatic dissemination was confirmed, although catecholamines and chromogranin levels were decreasing compared with the previous measures. Treatment was kept the same for 6 cycles of temozolomide. In December 2016, most of lesions had disappeared according to PET-TAC (Figure 1E). The two remaining lesions had maximum SUV significantly decreased. In January 2017, lanreotide was retired due to adverse side effects (as before).

In July 2017, a new PET supported the previous results, showing the remaining two lesions but with maximum SUV lower (Figure 1F). Temozolomide was withdrawn because of side effects (lymphopenia and thrombopenia). In October 2017, 4 months since the last dose of temozolomide, and propranolol as the only treatment (240mg/day), the disease remains controlled according to the last PET-TAC (F18-FDG) (Figure 1G). This imaging study suggests the presence of two neoplastic bone lesions in the dorsal spine and right acetabulum; regarding the previous examination (July 2017), the lesions described in D6 and acetabulum persist without major changes, whereas that of vertebra D10 has disappeared.

\section{Discussion}

The present case report describes an impressive clinical and metabolic improvement of a patient suffering from a metastatic paraganglioma after using a combination of temozolomide and propranolol at high doses. The improvement has been kept even after 7 months of lanreotide removal (usually used as standard treatment).

We can only hypothesize about the reason for this spectacular result. However, there are some literature reports on the synergy between chemotherapeutic agents inducing DNA damage, and therefore blocking DNA replication (as it is here the case of temozolomide) and $\beta$-blockers, which have shown antiangiogenic and pro-apoptotic effects in tumoral cells ${ }^{9,10}$.

Recent examples of these combinations found in the literature include the action of $\beta$-blockers increasing response to different chemotherapeutic agents. Pasquier et al. used a mouse model of neuroblastoma, where $\beta$-blockers were shown to increase treatment efficacy ${ }^{11}$. Propranolol in combination with vinblastine (chemotherapeutic agent belonging to the group of vegetable alkaloids), proved to be a successful treatment for advanced angiosarcoma ${ }^{12}$, with optimal results in seven patients following a clinical trial ${ }^{13}$. Chow et al. showed the regression of a rapidly enlarging stage T2 angiosarcoma of the scalp and face, following combination therapy with propranolol hydrochloride, paclitaxel (other vegetable alkaloid), and radiotherapy ${ }^{14}$. More recently, a prospective study using propranolol for off-label treatment of patients with melanoma, confirmed a significantly inversely association with recurrence of melanoma when propranolol was used at the time of diagnosis ${ }^{15}$. We could infer that propranolol seems to potentiate the anti-angiogenic effects and antitumor efficacy of chemotherapy.
Focusing our attention to propranolol- an unspecific $\beta$-blocker - as an antitumoral agent, Albiñana and coworkers ${ }^{16}$ recently published the clinical and biomarker follow-up of a clinical trial conducted in seven VHL patients with retinal hemangioblastomas. These patients had no surgical option, and the tumors remained stable in size (with reabsorption of exudates) following $120 \mathrm{mg}$ propranolol treatment for 12 months. This clinical trial led recently to the European Medicines Agency's designation of propranolol as Orphan drug for VHL disease.

The hypothesis supporting the antiangiogenic properties of propranolol relies on its proven effect on decreasing HIF-inducible transcription targets 9 . Propranolol would decrease HIF protein levels, and therefore, the activation of the hypoxia program developed by the hypoxia target genes, among them, angiogenic factors, such as VEGF, FGF, PDGF, EPO, and metalloproteases, activated in tumors that favor migration and dissemination of cancer cells.

The advantages of the use of propranolol and temozolomide derive mainly from the long experience as therapeutic agents. Both are old drugs, and therefore, the safety profile and side effects are well known. In this context, we can state that if they are proven effective in tumoral cases difficult to manage, the therapeutic solution may be immediate from the bench to the patient. In our case, after 4 months taking propranolol as the only medication, the improvement is maintained, which would support the use of propranolol as a drug that significantly increases the progression-free survival in absence of temozolomide. If these results are confirmed in more studies, its use would represent an advantage, allowing - once the disease is in remission - periods of time for the marrow to recover from the adverse effects of chemotherapy with the alkylating agent.

The strengths of the treatment are the results and the absence of serious side effects for the propranolol in this particular case. The limitations of using propranolol derive from the anti-hypertensive effects. Hypotensive patients should take care with the treatment, and be always under cardiologist supervision.

For these rare diseases where therapeutic options are scarce and with not very successful results, outcomes like the one reported here have to be seriously considered if we want to improve the quality and life expectancy for these - in most cases - young patients.

\section{Consent}

Written informed consent for the publication of the patient's clinical details and images was obtained from the patient.

\section{Author contributions \\ MD-C, KVGH, VA and L-MB wrote the paper. MD-C, TD-R and EG-F are the physicians responsible for the patient.}

\section{Competing interests}

No competing interests were disclosed.

\section{Grant information}

The author(s) declared that no grants were involved in supporting this work. 
1. Lenders JW, Duh QY Eisenhofer G, et al: Pheochromocytoma and Paraganglioma: An Endocrine Society Clinical Practice Guideline. J Clin Endocrinol Metab. 2014; 99(6): 1915-1942. PubMed Abstract | Publisher Full Text

2. Rednam SP, Erez A, Druker $\mathrm{H}$, et al.: Von Hippel-Lindau and hereditary pheochromocytoma/paraganglioma syndromes: Clinical features, genetics, and surveillance recommendations in childhood. Clin Cancer Res. 2017; 23(12): e68-e75.

PubMed Abstract | Publisher Full Text

3. Henegan JC Jr, Gomez CR: Heritable Cancer Syndromes Related to the Hypoxia Pathway. Front Oncol. 2016; 6: 68.

PubMed Abstract | Publisher Full Text | Free Full Text

4. Semenza GL: Oxygen homeostasis. Wiley Interdiscip Rev Syst Biol Med. 2010 2(3): 336-61.

PubMed Abstract | Publisher Full Text

5. Dhani N, Fyles A, Hedley D, et al.: The clinical significance of hypoxia in human cancers. Semin Nucl Med. 2015; 45(2): 110-21.

PubMed Abstract | Publisher Full Text

6. Taïeb D, Pacak K: New Insights into the Nuclear Imaging Phenotypes of Cluster 1 Pheochromocytoma and Paraganglioma. Trends Endocrinol Metab. 2017: 28(11): 807-817.

PubMed Abstract | Publisher Full Text | Free Full Text

7. Selak MA, Durán RV, Gottlieb E: Redox stress is not essential for the pseudohypoxic phenotype of succinate dehydrogenase deficient cells. Biochim Biophys Acta - Bioenerg. 2006; 1757(5-6): 567-72. PubMed Abstract | Publisher Full Text

8. Miettinen M, Killian JK, Wang ZF, et al: Immunohistochemical loss of succinate dehydrogenase subunit $A$ (SDHA) in gastrointestinal stromal tumors (GISTs) signals SDHA germline mutation. Am J Surg Pathol. 2013; 37(2): 234-40. PubMed Abstract | Publisher Full Text | Free Full Text
9. Albiñana V Villar Gómez de Las Heras K, Serrano-Heras G, et al: Propranolol reduces viability and induces apoptosis in hemangioblastoma cells from von Hippel-Lindau patients. Orphanet J Rare Dis. 2015; 10(1): 118. PubMed Abstract | Publisher Full Text | Free Full Text

10. Albiñana V, Recio-Poveda L, Zarrabeitia R, et al.: Propranolol as antiangiogenic candidate for the therapy of hereditary haemorrhagic telangiectasia. Thromb Haemost. 2012; 108(1): 41-53.

PubMed Abstract | Publisher Full Tex

11. Pasquier E, Street J, Pouchy C, et al: B-Blockers Increase Response To Chemotherapy Via Direct Antitumour and Anti-Angiogenic Mechanisms in Neuroblastoma. Br J Cancer. 2013; 108(12): 2485-94.

PubMed Abstract | Publisher Full Text | Free Full Text

12. Pasquier E, André N, Street J, et al:: Effective Management of Advanced Angiosarcoma by the Synergistic Combination of Propranolol and Vinblastinebased Metronomic Chemotherapy: A Bench to Bedside Study. EBioMedicine. 2016; 6: 87-95.

PubMed Abstract | Publisher Full Text | Free Full Text

13. Jenkins K: Propranolol in Angiosarcoma: First Major Advance in Decades. Medscape. 2017.

Reference Source

14. Chow W, Amaya CN, Rains S, et al.: Growth Attenuation of Cutaneous Angiosarcoma With Propranolol-Mediated $\beta$-Blockade. JAMA dermatology. 2015; 151(11): 1226-1229.

PubMed Abstract | Publisher Full Text

15. De Giorgi V, Grazzini M, Benemei S, et al.: Propranolol for Off-label Treatment of Patients With Melanoma Results From a Cohort Study. JAMA Oncol. 2017. PubMed Abstract | Publisher Full Text

16. Albiñana V, Escribano RMJ, Soler I, et al.: Repurposing propranolol as a drug fo the treatment of retinal haemangioblastomas in von Hippel-Lindau disease. Orphanet J Rare Dis. 2017; 12(1); 122.

PubMed Abstract | Publisher Full Text | Free Full Text 


\section{Open Peer Review}

\section{Current Peer Review Status:}

\section{Version 1}

Reviewer Report 21 February 2018

https://doi.org/10.5256/f1000research.14305.r30292

(C) 2018 Jara C. This is an open access peer review report distributed under the terms of the Creative Commons Attribution License, which permits unrestricted use, distribution, and reproduction in any medium, provided the original work is properly cited.

\section{Carlos Jara}

Alcorcón Foundation University Hospital, King Juan Carlos University (URJC), Madrid, Spain

I suggest to consider this work for indexing.

I think the subject is interesting, the presentation is appropriate and the arguments in favor of the advantage provided by propranolol are consistent. The mechanism by which this drug adds therapeutic advantage in this type of tumor remains an enigma, but I think that as a clinical case the arguments presented are sufficient.

The description of the case is correct and detailed. The clinical evolution is surprising and the role that propranolol can exercise together with temozolamide is promising and frankly novel. The clinical improvement and its maintenance over time are striking, particularly after the failure to previous standard therapies.

An especially positive feature of this study is to focus on a relatively rare pathology and in which the therapeutic possibilities are limited.

For a definitive evaluation of the role that propranolol may have in the treatment of these neoplasms, together with chemotherapy, it will be necessary to develop a multicenter phase III study, which, judging by the data provided by the authors, seems to be appropriate. It is of course necessary to investigate the mechanism by which propranolol potentiate the anti-angiogenic effects and antitumor efficacy of chemotherapy.

Suggestions for the authors:

At the beginning the phrase "Hereditary paraganglioma and pheochromocytoma syndromes (HPP) are characterized by rare tumors called pheochromocytomas (PHEOs) and paragangliomas (PGLs)." Should be reversed by "Hereditary paraganglioma and pheochromocytoma syndromes (HPP) are characterized by rare tumors called paragangliomas (PGLs) and pheochromocytomas (PHEOs).

In the description of the case it should be mentioned that the study carried out presumably 
was an 8F-DG-PET (no data is provided other than the SUV value).

I would suggest modifying "National Institutes of Health" by its name in Spanish, mentioning the specific hospital center.

I would suggest modifying the expression 'spectacular' by a more restrained one as 'striking'.

Is the background of the case's history and progression described in sufficient detail? Yes

Are enough details provided of any physical examination and diagnostic tests, treatment given and outcomes?

Yes

Is sufficient discussion included of the importance of the findings and their relevance to future understanding of disease processes, diagnosis or treatment?

Yes

Is the case presented with sufficient detail to be useful for other practitioners?

Yes

Competing Interests: No competing interests were disclosed.

I confirm that I have read this submission and believe that I have an appropriate level of expertise to confirm that it is of an acceptable scientific standard.

Reviewer Report 12 February 2018

https://doi.org/10.5256/f1000research.14305.r30293

(C) 2018 Juarranz A. This is an open access peer review report distributed under the terms of the Creative Commons Attribution License, which permits unrestricted use, distribution, and reproduction in any medium, provided the original work is properly cited.

\section{Angeles Juarranz}

Department of Biology, Autonomous University of Madrid, Madrid, Spain

The present case report describes an impressive clinical improvement of a patient suffering from a metastatic paraganglioma. The case is well written and documented with images. The good news is that the treatment is a combination of temozolomide and propranolol at high doses, old drugs from which the safety profile and side effects are well known.

It is noteworthy that the improvement was kept even after 7 months of lanreotide removal. Lanreotide remains one of the choice drugs as standard treatment for metastatic paragangliome, although with high side effects, and only limited success. 
While temozolomide is commonly used for brain tumors, the novelty is the concomitant treatment with a beta blocker, propranolol. The authors explain the results based on the antiangiogenic properties of propranolol, decreasing HIF-inducible transcription targets according to previous papers published elsewhere. Propranolol would decrease HIF protein levels, and thus the activation of hypoxia target genes, among them, angiogenic factors, such as VEGF, FGF, PDGF, EPO, and metalloproteases.

The advantages of the use of propranolol and temozolomide derive mainly from the long experience as therapeutic agents. Both are old drugs, and therefore, the safety profile and side effects are well known. In this context, we can state that if they are proven effective in tumoral cases difficult to manage.

For rare diseases where therapeutic options are scarce and with not very successful results, outcomes like the one reported here have to be seriously considered and spread in literature, since patients may get an enormous benefit. On top of this, the proposed treatment is cheap, and with minimal side effects.

However, this is a case report, and to be sure that the treatment really is effective, we need more knowledge derived from other cases.

Is the background of the case's history and progression described in sufficient detail? Yes

Are enough details provided of any physical examination and diagnostic tests, treatment given and outcomes?

Yes

Is sufficient discussion included of the importance of the findings and their relevance to future understanding of disease processes, diagnosis or treatment?

Yes

Is the case presented with sufficient detail to be useful for other practitioners? Yes

Competing Interests: No competing interests were disclosed.

I confirm that I have read this submission and believe that I have an appropriate level of expertise to confirm that it is of an acceptable scientific standard. 
The benefits of publishing with F1000Research:

- Your article is published within days, with no editorial bias

- You can publish traditional articles, null/negative results, case reports, data notes and more

- The peer review process is transparent and collaborative

- Your article is indexed in PubMed after passing peer review

- Dedicated customer support at every stage

For pre-submission enquiries, contact research@f1000.com 